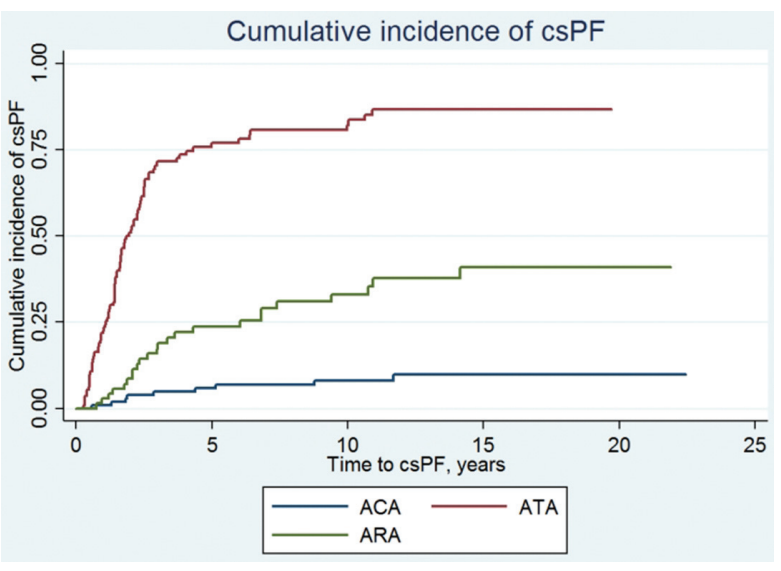

Conclusions: Our analysis demonstrates that csPF is a complication that tends to develop early in the disease course. Although the overall risk of csPF differs by antibodies, it is highest at around 3 years from disease onset and goes down thereafter. This can be used to inform organ disease monitoring and clinical trials recruitment.

Disclosure of Interest: None declared

DOI: 10.1136/annrheumdis-2018-eular.7089

\section{SAT0479 LASER SPECKLE CONTRAST ANALYSIS: A PILOT STUDY AND SYSTEMATIC LITERATURE REVIEW OF RELIABILITY OF THE QUANTITATIVE ASSESSMENT OF PERIPHERAL BLOOD PERFUSION IN SYSTEMIC SCLEROSIS}

A. Vanhaecke ${ }^{1,2}$, M. Cutolo $^{3}$, B. Ruaro ${ }^{3}$, L. Deroo ${ }^{1}$, C. Ickinger $^{4}$, K. Melsens ${ }^{1,2}$ Y. Piette ${ }^{2}$, F. De Keyser ${ }^{1,2}$, V. Smith ${ }^{1,2}$ on behalf of the EULAR study group on microcirculation in Rheumatic diseases. ${ }^{1}$ Department of Internal Medicine, Ghent University; ${ }^{2}$ Department of Rheumatology, Ghent University Hospital, Ghent, Belgium; ${ }^{3}$ Research Laboratory And Academic Division Of Clinical Rheumatology, Department Of Internal Medicine, Irccs San Martino Aou, University Of Genoa, Genoa, Italy; ${ }^{4}$ Division of Rheumatology, Faculty of Health Sciences, University of the Witwatersrand, Johannesburg, South Africa

Background: Microvasculopathy is an important feature of systemic sclerosis (SSc), making its assessment a key issue in SSc clinical research. Nailfold videocapillaroscopy (NVC) is a valuable tool to detect and classify microvascular structural alterations. In contrast, laser speckle contrast analysis (LASCA), a noninvasive microvascular imaging tool, has been proposed as an objective technique to dynamically evaluate the peripheral blood perfusion (PBP).

Objectives: The specific objectives were 1) to perform a pilot study to investigate as first both the intra- and inter-rater reliability of LASCA in an unselected SSc cohort and descriptively in healthy subjects (HS) and 2) to identify the available literature on the reliability of LASCA in SSc by a systematic literature review.

Methods: First, a pilot study was performed to assess the reliability of LASCA to measure the PBP at the level of the fingers in an unselected cohort SSc patients and descriptively in HS. Intra-rater reliability was assessed by having a first anchor rater performing the measurements at 2 time-points (within $15 \mathrm{~min}$ ) and inter-rater reliability by subsequently having the first anchor rater and a team of $3 \mathrm{~s}$ raters performing the measurements in $15 \mathrm{SSc}$ and $30 \mathrm{HS}$ (see figure 1). As external validation, the measurements were repeated with a second anchor rater in a distinct cohort of 15 SSc patients. Reliability was described by calculating the intraclass correlation coefficient (ICC).

The systematic search was performed to identify relevant full-text articles in PubMed, EMBASE and Web of Science. All retrieved articles were screened on title, abstract and full-text level, reference lists were additionally searched.

Results: Thirty SSc patients ( 5 men, 25 women; mean age $52 \pm 17$ y; 1 LSSc, 25LcSSc, 4DcSSc; 14 vasodilatory therapy) and $30 \mathrm{HS}$ (8 men, 22 women; mean age $33 \pm 11 \mathrm{y}$ ) underwent LASCA measurements. ICC for intra-rater reliability of the first anchor was $0.95(95 \% \mathrm{Cl} 0.86-0.98)$ in SSc and $0.93(95 \% \mathrm{Cl} 0.83-0.97)$ in HS, the ICC for inter-rater reliability was $0.97(95 \% \mathrm{Cl} 0.90-0.99)$ in SSc and 0.93 in HS. Intra- and inter-rater reliability of anchor 2 was $0.78(95 \% \mathrm{Cl} 0.46-$ $0.92)$ and $0.87(95 \% \mathrm{Cl} 0.67-0.96)$ respectively.

The systematic search identified 64 unique articles, of which 12 were eligible for full-text review. Two additional references were identified through a reference search of retrieved articles. Only 1 of the 14 selected references that met the inclusion criteria documented reliability as outcome and was included in the final analysis. This pilot study by Lambrecht et al measured the PBP at the level of the fingertips and reported ICC values varying from $0.82-0.91$ for the dorsal and $0.74-0.86$ for the volar fingertips.

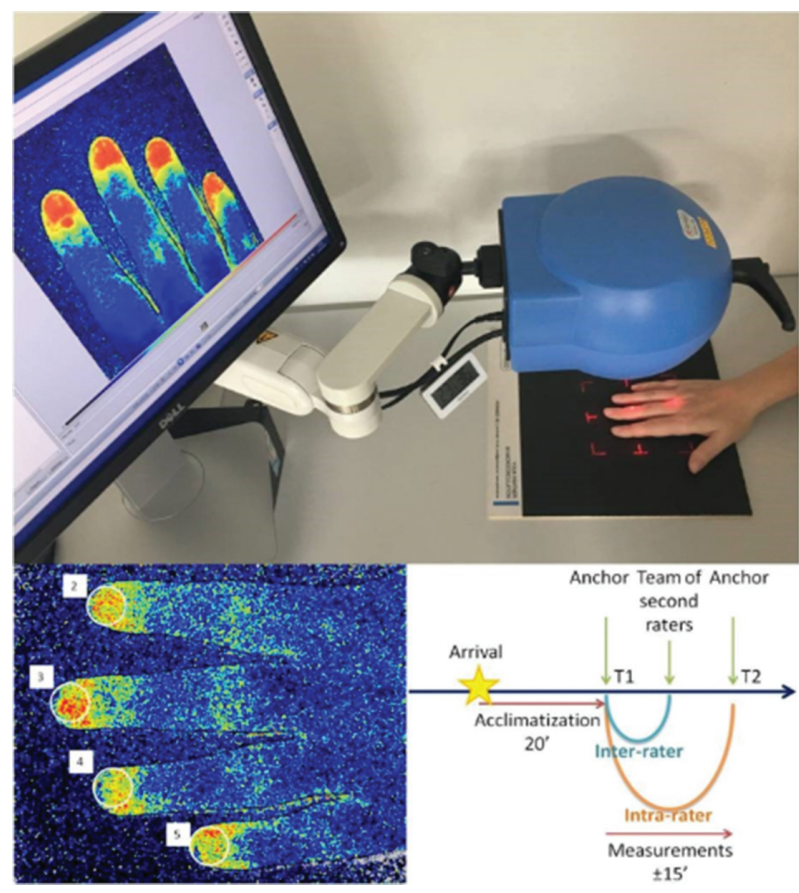

Conclusions: The body of knowledge regarding intra- and inter-rater reliability of LASCA in SSc is very limited. Only one manuscript reporting very good inter-rater reliability of PBP measurements of the distal fingertips by LASCA could be withheld. These results could be confirmed by our pilot study. In addition, we demonstrated excellent intra-rater reliability of LASCA measurements for the evaluation of the PBP of the hands in SSc patients and HS.

Disclosure of Interest: None declared

DOI: 10.1136/annrheumdis-2018-eular.3953

\section{SAT0480 EVALUATION OF STANDARDISED TEACHING OF MODIFIED RODNAN SKIN SCORE ASSESSMENT IN SYSTEMIC SCLEROSIS}

A.H. Low ${ }^{1,2}$, S.-A. $\mathrm{Ng}^{1,2}$, V. Berrocal ${ }^{3}$, B. Brennan ${ }^{3}$, G. Chan 4 , S.-C. Ng ${ }^{1,2}$, D. Khanna ${ }^{5} .{ }^{1}$ Rheumatology and Immunology, Singapore General Hospital; ${ }^{2}$ DukeNational University of Singapore, Singapore, Singapore; ${ }^{3}$ Biostatistics, University of Michigan, Michigan, USA; ${ }^{4}$ Rheumatology, Allergy and Immunology, Tan Tock Seng Hospital, Singapore, Singapore; ${ }^{5}$ Scleroderma Program, University of Michigan, Michigan, USA

Background: The modified Rodnan skin score (mRSS) is a standard outcome measure for skin involvement in systemic sclerosis (SSc) clinical trials. Training assessors reduces variability in mRSS measurement.

Objectives: Our objective is to report the inter- and intra-observer variability of mRSS scoring using newly developed standardised training guidelines by the Scleroderma Clinical Trials Consortium (SCTC).

Methods: Two SSc experts (DK/AL), 2 facilitators, 52 rheumatology trainees and 8 SSc patients fulfilling the 2013 American College of Rheumatology criteria participated in a SSc skin scoring workshop. Eight SSc patients were examined by 2 SSc experts and facilitators together and consensus scores reached. All trainees attended a talk on mRSS skin scoring by an SSc expert (DK), followed by a video and live demonstration by an expert examining a patient exhibiting different aspects of skin scoring. Each trainee subsequently performed mRSS scoring on 4 SSc patients independently. This concluded the teaching session for mRSS scoring. The mRSS scoring for each trainee was compared to the consensus expert mRSS, and a score of $\leq 5$ in 3 out of 4 patients is considered acceptable interobserver variability, as determined by SCTC guidelines.

Two days after training, 12 trainees, 2 facilitators and 2 experts re-assessed independently the mRSS of $2 \mathrm{SSc}$ patients whom they had examined previously. The repeat day $2 \mathrm{mRSS}$ score for each trainee was compared to the baseline mRSS score, and a score of $\leq 3$ is considered acceptable intra-observer variability.

We computed the inter- and intra-observer variability using a linear mixed model with an intercept term and random effects for patient, rater and patient by rater with the following values representing the degrees of agreement: $<0-$ poor; $0-0.20$ -slight; 0.21 - 0.40 -fair; $0.41-0.60$-moderate; $0.61-0.80$-substantial; and 0.81 1.00 -almost perfect agreement.

Results: For the first group of assessors involving 52 trainees, $65.4 \%$ of them achieved acceptable inter-observer variability, with inter-observer variability of 